\title{
Correlation between AHI and right ventricular diastolic function in patients with obstructive sleep apnea syndrome
}

\author{
Junfang $\mathrm{Li}^{1}$, Lu Changhong ${ }^{1}$, and Zhibing Wang ${ }^{2}$ \\ ${ }^{1}$ Affiliation not available \\ ${ }^{2}$ Qingdao University Affiliated Hospital
}

June 8,2020

Correlation between AHI and right ventricular diastolic function in patients with obstructive
sleep apnea syndrome Junfang $\mathrm{Li}^{1}, \mathrm{MD}, \mathrm{PhD}$, Changhong $\mathrm{Lu}^{2}$, MD, Zhibin Wang ${ }^{1}, \mathrm{MD}, \mathrm{PhD}$

1, Department of Echocardiography, Qingdao University Affiliated Hospital, Qingdao, 266001, China

2, Department of Heart Center, Qingdao Fuwai Cardiovascular Hospital, Qingdao, 266034,China

Corresponding author: Zhibin Wang, Department of Echocardiography, Qingdao UniversityAffiliated Hospital, No. 16, Jiangsu Road, Qingdao 266001, China

\section{Co-authors}

JunfangLi, MD, PhD.E-mail: qdljf @126.com

Changhong Lu, MD. E-mail: luch7879@126.com

Zhibin Wang, MD, PhD.E-mail:18661801291@163.com

Running Head Correlation between AHI and RV function inOSAS

Key Words 2D Echocardiography, Right ventricular function, Obesity, Pulmonary hypertension

Thank you very much for your attention to our article. You mentioned the relationship between obesity and pulmonary hypertension [1], and we also paid attention to the relevant studies. We agree with you that obesity usually occurs earlier than obstructive sleep apnea syndrome(OSAS).

The purpose of our study is to assess the influence of OSAS on right ventricular (RV) diastolic dysfunction[2]. Therefore, in order to eliminate the influence of obesity on $\mathrm{PH}$, in addition the poor image quality of obese patients, we reduce the inclusion of obese patients as far as possible.Furthermore,in order to eliminate the interaction among factors such as hypertension, body mass index (BMI) and elevated SPAP, multivariate linear regression analysis was used to determine whether severe OSAS was an independent parameter for early impaired RV diastolic function. The results showed that AHI right ventricular function were still negatively correlated.

Multivariate linear regression analysis of RV functional indices with AHI

\begin{tabular}{llllll}
\hline & Coef. & Std. Error & Beta & $t$ & $P$ \\
\hline RV-SRe & -0.516 & 0.258 & -0.281 & -2.495 & 0.026 \\
\hline
\end{tabular}


In our research, we mainly studied the influence of one factor of OSAS, AHI, on RV diastolic function.However, OSAS is actuallya disease combined with multiple factors. We look forward to further clinical research on OSAS.

\section{REFERENCES}

1.Berg G, Delaive K, Manfreda J, Walld R, Kryger MH. The use of health-care resourcesin obesity- hypoventilation syndrome. Chest 2001,120(2):377-383.

2.Li J, Lin X, Li H, Lu C, Li R, Liu W, Wang Z. Right ventricular diastolic dysfunction in patients with obstructive sleep apnea syndrome. Echocardiography. 2020Feb;37(2):317- 322. 